\title{
Toward Attogram Mass Measurements in Solution with Suspended Nanochannel Resonators
}

\author{
Jungchul Lee, ${ }^{\dagger}$ Wenjiang Shen, ${ }^{\ddagger}$ Kris Payer, ${ }^{\S}$ Thomas P. Burg, ${ }^{*}$, Il and Scott R. Manalis ${ }^{*},+\perp$ \\ †Department of Biological Engineering, Massachusetts Institute of Technology, Cambridge, Massachusetts 02139 , \\ ${ }^{*}$ Innovative Micro Technology, Santa Barbara, California 93117, ${ }^{\S}$ Microsystems Technology Laboratories, \\ Massachusetts Institute of Technology, Cambridge, Massachusetts 02139, "Max Planck Institute for Biophysical \\ Chemistry, 37077 Göttingen, Germany, and ${ }^{\perp}$ Department of Mechanical Engineering, Massachusetts Institute of \\ Technology, Cambridge, Massachusetts 02139
}

\begin{abstract}
Using suspended nanochannel resonators (SNRs), we demonstrate measurements of mass in solution with a resolution of $27 \mathrm{ag}$ in a $1 \mathrm{kHz}$ bandwidth, which represents a 100-fold improvement over existing suspended microchannel resonators and, to our knowledge, is the most precise mass measurement in liquid today. The SNR consists of a cantilever that is $50 \mu \mathrm{m}$ long, $10 \mu \mathrm{m}$ wide, and $1.3 \mu \mathrm{m}$ thick, with an embedded nanochannel that is $2 \mu \mathrm{m}$ wide and $700 \mathrm{~nm}$ tall. The SNR has a resonance frequency near $630 \mathrm{kHz}$ and exhibits a quality factor of approximately 8000 when dry and when filled with water. In addition, we introduce a new method that uses centrifugal force caused by vibration of the cantilever to trap particles at the free end. This approach eliminates the intrinsic position dependent error of the SNR and also improves the mass resolution by increasing the averaging time for each particle.
\end{abstract}

KEYWORDS Cantilever, mass sensor, nanochannel resonator, nanoelectromechanical systems, suspended microchannel resonator

( ver the past decade, significant progress has been made toward the advancement of mechanical resonators that can directly measure the mass of nanoscale particles and single molecules. ${ }^{1-8}$ Recently, innovative approaches to fabricating nanomechanical structures weighing $10^{-21} \mathrm{~kg}^{9-11}$ as well as high-resolution readout techniques have allowed measurements of mass with a noise floor below $10^{-25} \mathrm{~kg}$ in a $1 \mathrm{~Hz}$ bandwidth-less than the mass of a single gold atom. ${ }^{11}$ With further progress in miniaturization and the development of improved detection technology, it is conceivable that single-proton level resolution can ultimately be achieved, giving access to a wide range of applications. Arrays of such devices could, for example, be used for direct mass spectrometry of biological molecules without the need for electrostatic fragmentation. ${ }^{12}$ Since the mass of the resonator and the mechanical quality factor, which is a measure of damping, are the most critical parameters for attaining high resolution, the most precise measurements so far have been achieved using nanomechanical devices operating in vacuum.

It is envisioned that nanomechanical resonators when operated in solution could be used to weigh individual nanoparticles, viruses, protein aggregates, and ultimately macromolecules. However precision measurement of mass in the aqueous environment has proved to be more chal-

* To whom correspondence should be addressed, scottm@media.mit.edu to S.R.M. and tburg@mpibpc.mpg.de to T.P.B.

Received for review: 03/29/2010

Published on Web: 06/08/2010 lenging due to the presence of viscous drag, which increases the effective mass and rapidly dissipates energy from the resonator, thus severely degrading frequency resolution. Micromechanical cantilever resonators in liquid, for example, typically exhibit quality factors of only 1-10 (compared to 1000-10000 for resonators in vacuum), and viscous damping becomes increasingly detrimental as the size of the resonator is decreased. ${ }^{13}$ Previously, we demonstrated that viscous loss could be nearly eliminated by placing the fluid inside of the resonator. This device, known as the suspended microchannel resonator (SMR), exhibited a high quality factor of 15000 and enabled single cells and single gold nanoparticles down to $100 \mathrm{~nm}$ in diameter to be weighed in a flow-through format. ${ }^{14}$ However, the suspended microchannel weighs $10^{-10} \mathrm{~kg}$, and with a frequency resolution of 10 parts-per-billion (ppb) in a $1 \mathrm{~Hz}$ bandwidth, the mass resolution is limited to approximately $10^{-18} \mathrm{~kg}$, or $5.0 \times 10^{-18} \mathrm{~kg}$ in a $1 \mathrm{kHz}$ bandwidth.

To further improve the mass resolution, we have reduced the mass of the SMR by 75 -fold by decreasing the cantilever length, width, and thickness. Importantly, we find that the quality factor remains at approximately 8000 with and without liquid inside the channel, as previously predicted by theory. ${ }^{15}$ This device, which we call the suspended nanochannel resonator (SNR), achieves a mass resolution of $2.7 \times$ $10^{-20} \mathrm{~kg}$ (or $27 \mathrm{ag}$ ) in a $1 \mathrm{kHz}$ bandwidth. We demonstrate that particles can either be weighed in a continuous flowthrough format as before or, alternatively, be sequentially trapped at the apex of the resonator by centrifugal force and 
subsequently removed by increasing the flow rate. The latter approach eliminates measurement error due to uncertainty in the transverse position of particles inside the channel when flowing through the turn at the apex of the cantilever. In addition, trapping allows for longer averaging times (typically several seconds).

The frequency shift induced by a particle near the free end of the resonator is given by $\Delta f l f \approx-\Delta m / 2 m_{\text {eff }}$ where $f$ is the resonance frequency and $m_{\text {eff }}$ is the effective mass of the resonator, respectively. Mass resolution can thus be most readily improved by making the resonator as light as possible while maintaining the capability to measure the resonance frequency with high precision. The latter is achieved by simultaneously maximizing the quality factor and the dynamic range of the vibration measurement, i.e., the ratio between the attainable vibration amplitude and the measurement noise. ${ }^{16}$ While miniaturization is a powerful route to lowering the mass of the resonator due to the cubic dependence of mass on linear size, a reduction in size is typically accompanied by a reduction in quality factor and an increase in fabrication complexity. In this work, we chose to use high-yield conventional microfabrication processes to make resonators with dimensions near the practical limit of photolithography-based microelectromechanical systems technology.

SNRs were batch-fabricated on 6 in. wafers at Innovative Micro Technology (Santa Barbara, CA). The fabrication process is similar to that of SMRs described previously. ${ }^{14}$ The resonators are $50 \mu \mathrm{m}$ long, $10 \mu \mathrm{m}$ wide, and $1.3 \mu \mathrm{m}$ thick cantilever beams with embedded nanofluidic channels which are $2 \mu \mathrm{m}$ wide and $700 \mathrm{~nm}$ tall. The total length of the buried nanochannel including the U-shaped channel inside the suspended resonator and straight channel on the solid support is $\sim 375 \mu \mathrm{m}$. An on-chip getter maintains a high vacuum in the cavity around the cantilever. Similar to existing SMRs, SNRs rely on electrostatic actuation and optical-lever detection. A thin reflective aluminum coating on the SNR improves the optical-lever sensitivity and minimizes heating by efficiently reflecting the readout laser. The SNR is approximately 75 -fold lighter than the current smallest SMR which is $210 \mu \mathrm{m}$ long, $33 \mu \mathrm{m}$ wide, and $7 \mu \mathrm{m}$ thick. The expected resonance frequency of the dry SNR is 784 $\mathrm{kHz}$ based on analytical calculations and $776 \mathrm{kHz}$ based on finite element analysis (FEA) using COMSOL Multiphysics. FEA predicts a spring constant of $7.09 \mathrm{~N} / \mathrm{m}$ for point loading at the tip and a mass sensitivity of $-1.35 \mathrm{~Hz} / \mathrm{fg}$. Electrostatic actuation is achieved by applying a drive signal between the cantilever (ground plane) and an electrode located approximately $25 \mu \mathrm{m}$ below the cantilever. Figure 1 a shows an optical micrograph of the final device and reveals two symmetric bypass channels, the buried nanofluidic channel running through the resonator and squared apertues $(3 \mu \mathrm{m}$ $\times 3 \mu \mathrm{m}$ ) connecting the embedded nanofluidic channel to each bypass channel. A scanning electron micrograph acquired before the resonator was released shows a cutaway
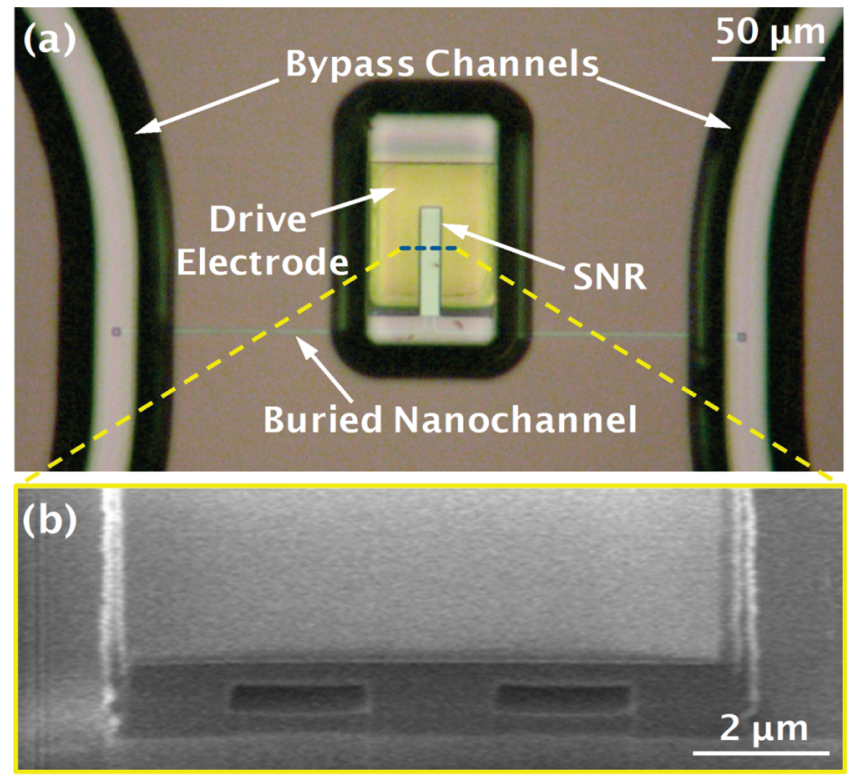

FIGURE 1. (a) Optical micrograph showing the fabricated suspended nanochannel resonator (SNR). (b) Scanning electron micrograph of a cutaway view of the SNR showing the buried nanofluidic channel, which is $2 \mu \mathrm{m}$ wide and $700 \mathrm{~nm}$ tall.

view of the SNR having an embedded nanochannel which is $2 \mu \mathrm{m}$ wide and $700 \mathrm{~nm}$ tall (Figure 1b). The nearly symmetric placement of the embedded fluidic channel about the neutral plane of the cantilever improves the quality factor when the resonator is filled with fluid by minimizing the pumping loss. ${ }^{15}$

To operate the SNR, the optical-lever detection setup orignally developed for the $\mathrm{SMR}^{14}$ is modified. The collimated output of a diode laser module (Coherent ULN, 635 $\mathrm{nm}$ ) is expanded to a waist diameter of $\sim 5 \mathrm{~mm}$ and sent through an iris diaphragm, polarizing beamsplitter, and a quarter wave plate before it is focused onto the cantilever by a $10 \times$ microscope objective $(10 \times, \mathrm{NA}=0.3, \mathrm{WD}=17.5$ $\mathrm{mm}$ ). The reflection is collected by the same objective and directed through the beamsplitter onto a segmented photodiode (SPOT-2D, UDT Sensors). This setup ensures that the focal spot is smaller than the width of the SNR. The dc bias for electrostatic actuation is fixed at $120 \mathrm{~V}$; we found no noticeable improvement in the frequency noise at higher bias voltages.

The frequency response characteristic of the resonator was measured with a lock-in amplifier (SR844, Stanford Research Systems) while the frequency of the ac excitation was swept at a fixed amplitude with a function generator (33220A, Agilent). Figure 2a shows the normalized amplitude responses near the fundamental resonance for the dry SNR and SNR filled with heavy water $\left(\mathrm{D}_{2} \mathrm{O}\right)$, water $\left(\mathrm{H}_{2} \mathrm{O}\right)$, and ethanol at room temperature. After each sample is measured, the SNR is flushed with water and dried with nitrogen to minimize errors resulting from incomplete fluid exchange. A damped simple harmonic oscillator model is used to extract the resonance frequency and quality factor. Since the 
(a)

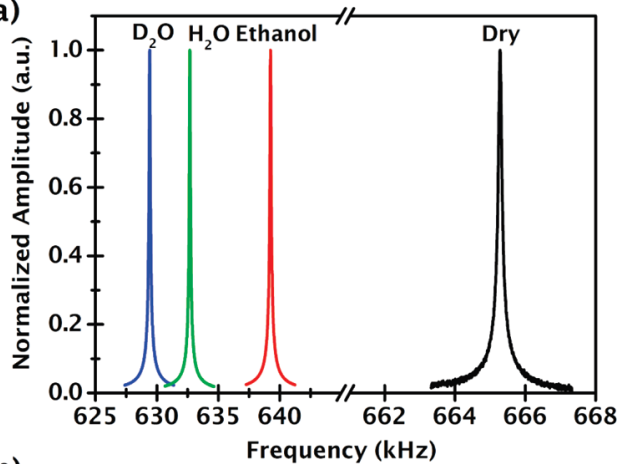

(b)

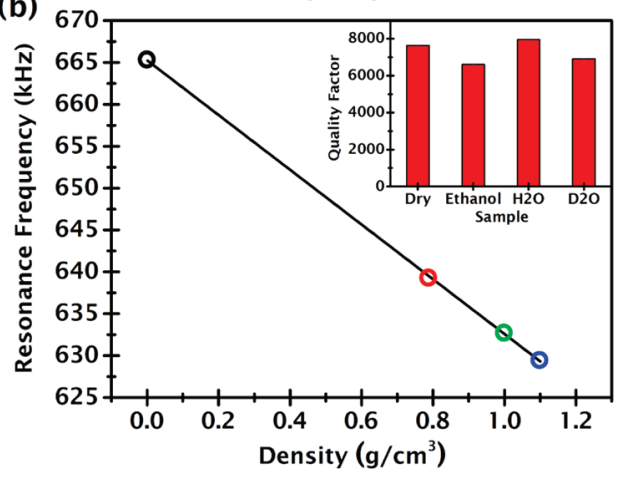

FIGURE 2. (a) Normalized amplitude responses near the fundamental resonance for the dry SNR and SNR filled with heavy water (density $\sim 1.1 \mathrm{~g} / \mathrm{mL})$, water $(\sim 1.0 \mathrm{~g} / \mathrm{mL})$, and ethanol $(\sim 0.8 \mathrm{~g} / \mathrm{mL})$ at room temperature. (b) Resonance frequency of the SNR decreasing linearly with fluid density (adjusted $R^{2}=0.9999$ for the linear fit). Inset shows quality factors of the dry SNR and SNR filled with liquid. The quality factor of the liquid-filled SNR is not significantly different from that of the dry SNR.

measured resonance frequency of $665.279 \pm 0.002 \mathrm{kHz}$ is lower than our theoretical estimation, it is possible that the actual SNR is slightly longer and/or thinner than the nominal design specifications. As expected, the resonance frequency decreases approximately linearly with the fluid density.

Measurements of the quality factor when the SNR is filled with air, ethanol, $\mathrm{H}_{2} \mathrm{O}$ or $\mathrm{D}_{2} \mathrm{O}$ are shown in the inset in Figure 2b. Interestingly, the quality factor of the wet SNR is not significantly lower than that of the dry SNR. Moreover, the quality factor of the SNR filled with water $(7954 \pm 16)$ is actually higher than that of the dry SNR $(7627 \pm 18)$. All data points were obtained in triplicate to ensure that the observed differences in the quality factor between different fluids were larger than the measurement error. The nonmonotonic dependence of the quality factor on fluid viscosity is consistent with theory and experiments conducted in SMRs of larger dimensions. ${ }^{15}$ Increasing viscosity can increase or decrease the amount of fluid-induced damping due to the complex interplay between different fluid dynamic regimes which govern dissipation in microfluidic cantilever resonators. When these devices are filled with air, dissipation is dominated by the intrinsic energy loss of the solid resonator. When filled with liquid, shear induced viscous dissipation acts to lower the quality factor, while the added mass of the solution increases the amount of energy stored in the vibrating beam, thereby providing a small increase in the quality factor. These effects can conspire to result in no measurable change or even an effective increase in quality factor when the cantilever is filled. The precise magnitude of viscous damping is a nontrivial function of the channel geometry, vibration frequency, and the properties of the fluid, ${ }^{15}$ which may explain the variations in the quality factor observed in our experiments.

Although each amplitude response is shown normalized with its maximum at resonance (Figure 2a), the absolute magnitudes were similar for both dry and wet SNRs. This is a further indication that the quality factor of the SNR is relatively insensitive to the presence of liquid. As a result, the freqeuncy noise of the liquid-filled SNR is similar to that of the dry SNR.

Measurements of resonance frequency as a function of time were obtained by incorporating the cantilever as the frequency determining element into an oscillator circuit. The circuit is formed by feeding back the phase shifted and amplified signal from the photodetector transimpedance amplifier to the dc-biased electrostatic drive electrode. The phase shifter is adjusted so that the accumulated phase of the cantilever and feedback path is $180^{\circ}$ at resonance. The oscillator thus continuously tracks the resonance frequency of the cantilever without requiring an external frequency source. The output of the oscillator is mixed down to a frequency in the kilohertz range using a function generator as reference oscillator (33220A, Agilent). The resulting frequency is then measured using a digital frequency counter (PCI-6259, National Instruments).

After the SNR was calibrated by measuring solutions of known densities, gold nanoparticles were introduced into the SNR and weighed. Gold nanoparticles (50 $\mathrm{nm}$ diameter) at an initial concentration of $4.5 \times 10^{10} / \mathrm{mL}$ (C-Au-0.050, Corpuscular Inc.) were diluted to $4.5 \times 10^{7} / \mathrm{mL}$ with deionized water and sonicated for 2 min to break apart any aggregates. When individual gold nanoparticles travel through the SNR, a downward peak is measured, as shown in Figure 3a (flow-through mode). The average transit time for each gold nanoparticle was $\sim 100 \mathrm{~ms}$ for a pressure differential of $\sim 10 \mathrm{kPa}$ between the sample and waste bypass channels. This transit time corresponds to a volumetric flow rate of $\sim 1 \mathrm{pL} / \mathrm{s}$. When minor losses originating from the four rounded sections (each is a $90^{\circ}$ elbow) in the nanochannel are neglected, this flow rate is consistent with the calculated Hagen-Poiseuille flow rate given the experimental conditions. ${ }^{17}$

When the pressure difference is reduced to $5 \mathrm{kPa}$, the volumetric flow rate and particle velocity decrease correspondingly. Interestingly, incoming particles become trapped at the tip of the resonator and result in a series of step changes in the resonance frequency (Figure 3b). The trapping effect is expected, since for slow flow velocities the Stokes drag may become smaller than the centrifugal force acting on a particle near the cantilever tip. This force can 
(a)

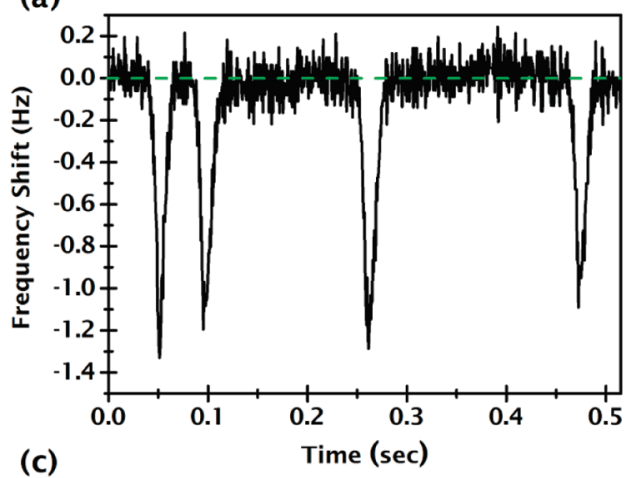

(c)

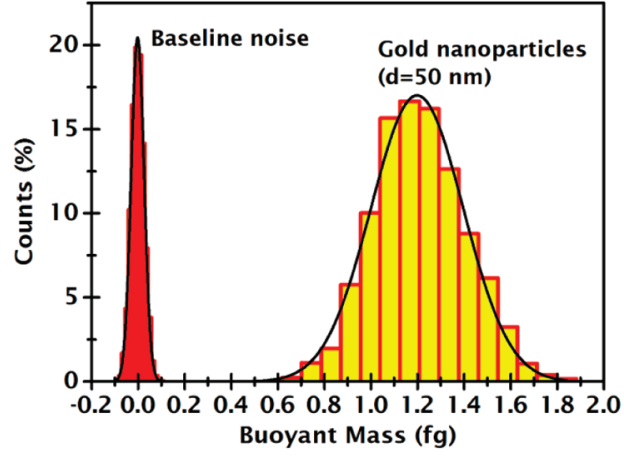

(b)

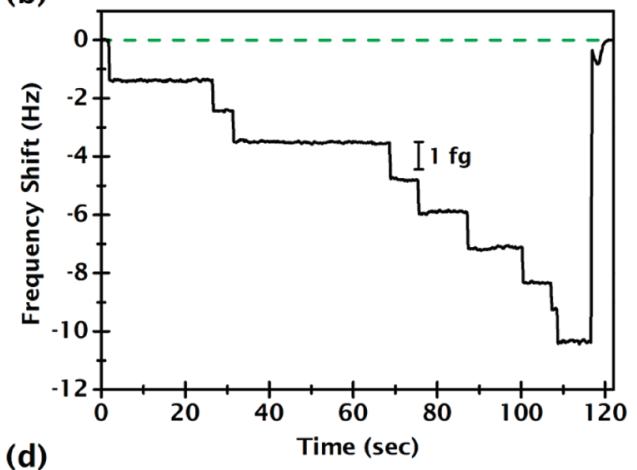

(d)

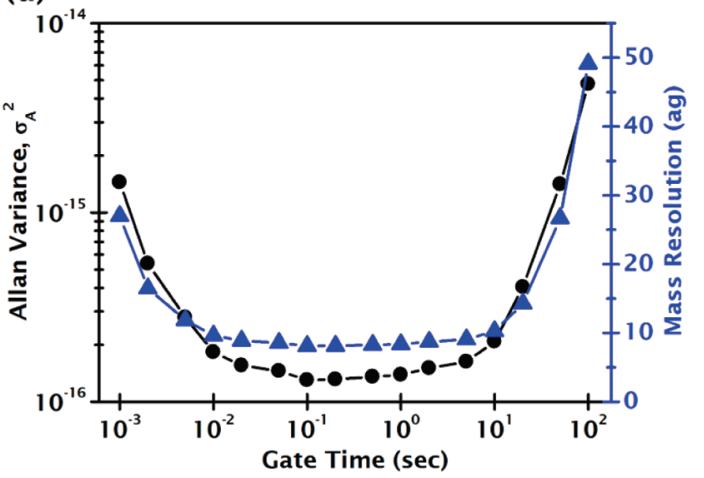

FIGURE 3. (a) Resonance frequency shift of the SNR when $50 \mathrm{~nm}$ diameter gold nanoparticles flow through the resonator. Raw data shown here are taken at a sampling rate of $1.5 \mathrm{kHz}$. (b) Resonance frequency shift of the SNR when gold nanoparticles are trapped at the apex of the resonator. Each step represents the trapping of a single $50 \mathrm{~nm}$ diameter gold nanoparticle. After nine particles are trapped, the flow direction is reversed to unload the trapped particles and the resonance frequency returns to its orginal value (at $t=118 \mathrm{~s}$ ). (c) Mass histograms corresponding to short-term baseline frequency noise (10 s of data, $1 \mathrm{~ms}$ averaging per data point) and frequency shift from a population of $50 \mathrm{~nm}$ diameter gold nanoparticles in the flow-through mode. (d) Allan variance in the measured frequency noise (circles) and mass resolution (triangles) as a function of gate time for a baseline sampled for $1 \mathrm{~h}$ at a rate of $1 \mathrm{kHz}$. Mass sensitivity for the flow-through mode is used to calculate mass resolution.

become very large under the conditions of our experiments, as we now explain. A first-order estimate for the axial acceleration near the cantilever tip is obtained by approximating the trajectory of the tip as an arc centered about the base of the cantilever. The instantaneous angular velocity is given by $\dot{\theta}=A / l 2 \pi f \sin (2 \pi f t)$, where $l$ is the length of the cantilever, $A$ is the vibration amplitude, and $f$ is the resonance frequency for the fundamental mode. At $3 \mu \mathrm{m}$ vibration amplitude, which we estimate based on the geometry and the applied drive voltage, the average centrifugal acceleration over one cycle is $\sim 1.6 \times 10^{6} \mathrm{~m} / \mathrm{s}^{2}$. A flow velocity of $\sim 4 \mathrm{~mm} / \mathrm{s}$ is required to balance this acceleration acting on a $50 \mathrm{~nm}$ gold particle in water; this is on the same order as the flows employed in our experiments. In general, the fluid velocity required for removing a trapped particle will be larger than the estimate above, since the particle can interact with the surface.

While we have also observed trapping of particles to occur in suspended microchannel resonators, significantly stronger excitation was required to observe the effect. This is expected due to the square dependence of the centrifugal force on angular velocity, which results in an approximately 100 times weaker trapping force in the $7 \mu \mathrm{m}$ thick and 210 $\mu \mathrm{m}$ long SMRs reported previously by our group. ${ }^{14}$
Each step shown in Figure 3b represents a single gold nanoparticle that becomes trapped. Data shown in this figure are sampled at $1 \mathrm{kHz}$, low pass filtered with a cutoff frequency of $50 \mathrm{~Hz}$, and then moving averaged with 250 adjacent points. Figure $3 \mathrm{~b}$ also shows that several sequentially trapped particles can be easily knocked out and the resonance frequency of the SNR can be reset to its original value when the flow is reversed for a few seconds with a high pressure difference. This inertial trapping and pressurized unloading step can be repeated and used to generate a histogram of particle masses (inertial trapping mode). Although the measurement throughput of the inertial trapping mode is lower than the flow-through mode, the signal averaging time for each particle can be controlled. More importantly, the intrinsic error originating from the uncertain transverse position of the particles inside the channel is eliminated. As a result, the minimum detectable mass dispersion for a particle population is governed by the mass resolution, which is less than $10 \mathrm{ag}$ in a $1 \mathrm{~Hz}$ bandwidth.

Figure $3 \mathrm{c}$ shows mass histograms corresponding to shortterm (10 s, $1 \mathrm{kHz}$ bandwidth) baseline frequency noise and the frequency shift measured from a population of $50 \mathrm{~nm}$ diameter gold nanoparticles $(N=1789)$ in the flow-through mode, also at a $1 \mathrm{kHz}$ bandwidth. The standard deviation 
TABLE 1. Standard Deviation in Measured Mass Histograms Resulting from Three Contributions: System Noise Level, Particle Distribution, and Particle Position

\begin{tabular}{lcc}
\hline $\begin{array}{c}\text { Standard } \\
\text { deviation (ag) }\end{array}$ & $\begin{array}{c}\text { flow-through } \\
\text { mode }\end{array}$ & $\begin{array}{c}\text { inertial trapping } \\
\text { mode }\end{array}$ \\
\hline system noise & 27 & 26 \\
particle distribution & 178 & 178 \\
particle position $^{a}$ & 87 & 180 \\
total $^{a}$ & 200 & \\
a Square root of sum of squares of each contribution.
\end{tabular}

of the baseline frequency noise is $38.6 \mathrm{ppb}$, and the mass sensitivity of $0.890 \mathrm{~Hz} / \mathrm{fg}$ is deduced from the size specification of the nanoparticles and the density of gold. The average and standard deviation of the measured gold nanoparticles are 1.20 and $0.20 \mathrm{fg}$, respectively, and the coefficient of variation (CV) in buoyant mass is $16.7 \%$. For comparison, the inertial trapping mode was applied to the same particle population. The mass sensitivity of $0.944 \mathrm{~Hz} / \mathrm{fg}$ is slightly higher in this mode since the position of each particle trapped is closer to the cantilever tip. For 817 particles measured, the average and standard deviation are 1.20 and 0.18 fg, respectively, and the CV is $15.0 \%$. The ratio between the two aforementioned mass sensitivities agrees within $0.2 \%$ with the ratio between frequency shifts that are calculated at the midpoint and outer edge of the channel. The CV measured by the flow-through mode consists of three independent contributions: system noise level, mass distribution of the particle population, and error resulting from the uncertainty in which streamline each particle is following. Each contribution can be determined by comparing the CVs from the baseline frequency noise and the flowthrough and inertial trapping modes (Table 1). For the 50 $\mathrm{nm}$ gold nanoparticles, the polydispersity of the particles is the dominant contribution. We anticipate that the inertial trapping mode will be useful for analyzing highly monodisperse nanoparticles samples.

To determine how noise depends on the duration of the averaging window, the Allan variances ${ }^{18}$ were calculated from a $1 \mathrm{~h}$ measurement of the baseline frequency noise in a $1 \mathrm{kHz}$ bandwidth which is segmented and averaged in a given gate time (Figure $3 \mathrm{~d}$ ). Allan variance can be converted to mass resolution since the mass sensitivity is known, giving a mass equivalent noise of 27 ag for a $1 \mathrm{~ms}$ gate time; this is similar to the standard deviation of the noise histogram shown in Figure 3c. The best mass resolution of 8 ag occurs at a gate time between 0.1 and $1 \mathrm{~s}$. There are three distinct regimes visible in the plot of Allan variance vs gate time. For long gate times of 10-100 s, Allen variance increases with increasing time. This may be attributed to noise sources inherent to our frequency measurement system, since similar trends are observed when the SNR and oscillator circuitry are replaced with a function generator. For intermediate gate intervals from $10 \mathrm{~ms}$ to $10 \mathrm{~s}$, we observe only a weak dependence of frequency noise on measurement time, with a maximum variation of less than a factor of 2

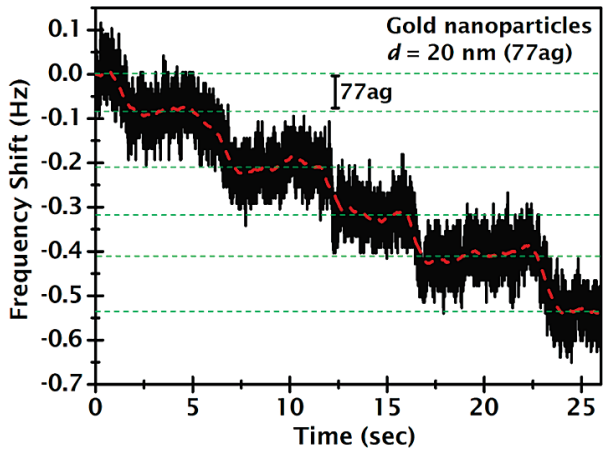

FIGURE 4. Resonance frequency shift of the SNR showing $20 \mathrm{~nm}$ diameter gold nanoparticles at a concentration of $3.5 \times 10^{9} / \mathrm{mL}$ are sequentially trapped at the apex of the resonator. Raw data shown (black solid line) are acquired at a sampling rate of $1 \mathrm{kHz}$, low pass filtered with a cutoff frequency of $50 \mathrm{~Hz}$, and then moving averaged with 1000 adjacent points (red dashed line).

over the entire range. For very short intervals (1 to $10 \mathrm{~ms}$ ), frequency resolution improves with increasing gate time. The flat region in the intermediate regime is likely the result of superposition between the opposing trends seen at extremes of short and long gate times. Stability of the oscillator is adversely affected by multiple factors, such as, for example, laser intensity noise, mechanical vibration, and small drift of circuit parameters, which may alter the phase shift in the feedback loop. Drift in alignment of the laser may also contribute to variation in the measured frequency on the time scale of minutes to hours. Since the thermomechanical frequency noise is at least 2 orders of magnitude lower than the noise level of our system, improvements in detection and drive circuitry may be as important for increasing mass resolution as further miniaturization of the resonator.

To demonstrate the high resolution of the SNR, we measured $20 \mathrm{~nm}$ diameter gold nanoparticles (C-Au-0.020, Corpuscular Inc.) which have a buoyant mass of $77 \mathrm{ag}$ in water. For comparison, an individual HIV virion has a buoyant mass of $\sim 80$ ag. ${ }^{19}$ Figure 4 shows the resonance frequency shift of the SNR for five sequential trapping events. The raw data (sampled at $1 \mathrm{kHz}$ ) are plotted in black and the filtered response is plotted with a red dashed line.

In this Letter, we introduced suspended nanochannel resonators and reported their performance. Our SNRs exhibit quality factors up to $\sim 8000$ and show little difference in quality factor between the dry and liquid-filled states. Using electrostatic excitation and optical-lever detection, we have achieved a frequency resolution of $39 \mathrm{ppb}$, corresponding to a mass resolution of $27 \mathrm{ag}$, in a $1 \mathrm{kHz}$ bandwidth. This constitutes a $\sim 100$-fold improvement over existing suspended microchannel resonators and, to the best of our knowledge, represents the most precise mass measurement achieved in liquid to date. Our results thereby lay the foundation for a wide array of precision measurements based on the direct detection of mass of single nanoscopic particles and minute quantities of surface-adsorbed mol- 
ecules. We believe that this technique will find numerous applications in fields ranging from biology and biophysics to materials science and nanotechnology. In addition, it can be expected that further miniaturization of devices using conventional batch microfabrication will yield another 10 fold improvement in mass sensitivity. While estimates indicate that the thermomechanical limit for mass resolution using these scaled suspended nanochannel resonators would reach well into the regime of single macromolecules, our results suggest that some of the key challenges to realizing this potential lie in a better implementation of the frequency measurement and associated instrumentation for detecting and exciting the vibration of the resonator.

Acknowledgment. This work was supported by CIMIT Contract 09-440 and the Institute for Collaborative Biotechnologies Grant (DAAD1903D0004) from the U.S. Army Research Office. T.P.B. acknowledges support by the Max Planck Society and the Max Planck Institute for Biophysical Chemistry. S.R.M. is a co-founder of Affinity Biosensors and declares competing financial interests.

\section{REFERENCES AND NOTES}

(1) Gupta, A.; Akin, D.; Bashir, R. Appl. Phys. Lett. 2004, 84 (11), 1976-1978.

(2) Ekinci, K. L.; Huang, X. M. H.; Roukes, M. L. Appl. Phys. Lett. 2004, 84 (22), 4469-4471.
(3) Ilic, B.; Yang, Y.; Craighead, H. G. Appl. Phys. Lett. 2004, 85 (13), 2604-2606.

(4) Ilic, B.; Craighead, H. G.; Krylov, S.; Senaratne, W.; Ober, C.; Neuzil, P. J. Appl. Phys. 2004, 95 (7), 3694-3703.

(5) Yang, Y. T.; Callegari, C.; Feng, X. L.; Ekinci, K. L.; Roukes, M. L. Nano Lett. 2006, 6 (4), 583-586.

(6) Peng, H. B.; Chang, C. W.; Aloni, S.; Yuzvinsky, T. D.; Zettl, A. Phys. Rev. Lett. 2006, 97 (8), No. 087203.

(7) Li, M.; Tang, H. X.; Roukes, M. L. Nat. Nanotechnol. 2007, 2 (2), $114-120$

(8) Feng, X. L.; He, R. R.; Yang, P. D.; Roukes, M. L. Nano Lett. 2007, 7 (7), 1953-1959.

(9) Lassagne, B.; Garcia-Sanchez, D.; Aguasca, A.; Bachtold, A. Nano Lett. 2008, 8 (11), 3735-3738.

(10) Chiu, H. Y.; Hung, P.; Postma, H. W. C.; Bockrath, M. Nano Lett. 2008, 8 (12), 4342-4346

(11) Jensen, K.; Kim, K.; Zettl, A. Nat. Nanotechnol. 2008, 3 (9), $533-$ 537.

(12) Naik, A. K.; Hanay, M. S.; Hiebert, W. K.; Feng, X. L.; Roukes, M. L. Nat. Nanotechnol. 2009, 4 (7), 445-450.

(13) Sader, J. E. J. Appl. Phys. 1998, 84 (1), 64-76.

(14) Burg, T. P.; Godin, M.; Knudsen, S. M.; Shen, W.; Carlson, G.; Foster, J. S.; Babcock, K.; Manalis, S. R. Nature 2007, 446 (7139), 1066-1069.

(15) Burg, T. P.; Sader, J. E.; Manalis, S. R. Phys. Rev. Lett. 2009, 102 (22), 228103

(16) Ekinci, K. L.; Yang, Y. T.; Roukes, M. L. J. Appl. Phys. 2004, 95 (5), 2682-2689.

(17) White, F. M. Fluid mechanics, 3rd ed.; McGraw-Hill: New York, 1994.

(18) Allan, D. W. Proc. IEEE 1966, 54 (2), 221

(19) Gallay, P.; Swingler, S.; Song, J. P.; Bushman, F.; Trono, D. Cell $1995,83(4), 569-576$. 